\title{
Study of Implicit Preferences in Facial Expression Recognition of Urban Internet-Addicted Left-Behind Children in China
}

\section{Ying $\mathrm{Ge}^{1,2 *}$, Jinfu Zhang ${ }^{1}$ and Yuanyan $\mathrm{Hu}^{2}$}

${ }^{1}$ Faculty of Psychology, Key Laboratory of Personality and Cognition, Ministry of Education, Southwest University, Beibei, Chongqing, China

${ }^{2}$ Laboratory of Cognition and Mental Health, Chongqing University of Arts and Sciences, Yongchuan, Chongqing, China

\begin{abstract}
For the purpose for exploring the differences in implicit preferences in facial expression recognition between urban internet-addicted left-behind children and urban left-behind children with no such addiction, variant GO/NO-GO (GNAT) paradigm of implicit association test (IAT) and a single-factor 2 level experimental design were adopted to test sixty participants (14 years of age) who were selected from two junior middle schools in Chongqing, China. This study results showed that compared with urban non-addicted left-behind children, urban Internet-addicted left-behind children gave more preference to negative emotion and poorer evaluation on their egos.
\end{abstract}

Keywords: Internet addiction; Urban left-behind children; Facial expression recognition; Implicit preference

\section{Introduction}

Emotional image recognition of facial expression is an important and widely used measure and method for study of emotions. Standardized emotional image system carries out matching of emotional valence and emotional arousal towards emotional stimulation materials, increases possibility of equal positive and negative intensity from emotion elicitation and makes experimental results possess higher comparability and stability. After emotion elicitation, signal detection theory is a general method to detect recognition condition of participants against experimental materials. Variant GO/NO-GO (Go/ No-go association test, GNAT) test of implicit association test (IAT) that is used to measure internal mental process of individuals against a kind of thing or phenomenon tests participants' hit rate and false alert rate under different unified tasks by adopting principles of signal detection theory. Response of participants to stimulus that represents target and attribute categories is named as hit rate (i.e. Go), and no response to other represented stimuli is referred to as false alert rate (i.e. No-go). The hit rate and false alert rate are converted to z-score and the difference value is taken as d'-score; then, through comparison of $\mathrm{d}^{\prime}$-score under different combinations of target concept and attribute concept, perceptibility, propensity and preference of participants are reflected [1].

In 1980s, China witnessed its social and economic transition period, and a large number of adults rushed from underdeveloped western regions to developed eastern regions for work opportunities. These adults left their children in native homes, bringing a group named "left-behind children" into existence. In the later period of 1990s, the left-behind children especially referred to rural children, below 18 years old, who were left in their rural native homes for over a half year and needed to be taken under tutelage of other adults because their mothers, fathers or both parents left for work and came back homes with an interval more than three months [2-9].

Due to various reasons, the number of urban left-behind children is increasing in recent years. There are nearly 20 million left-behind children based on results of the fifth population census, including approximate 2.7 million urban left-behind children, accounting for $13.5 \%$ of the total number [10]. As the only municipality directly under the central government in Western China, Chongqing has 1.07 million left-behind children, accounting for $34 \%$ of total number of students in compulsory education stage of the region [11].
Thus, with changes in meaning, left-behind children include urban left-behind children and rural left-behind children, and the urban leftbehind children have attracted attentions from all sides. The urban leftbehind children refer to children, below 18 years old, who live under cares or tutelage from nurses, grandparents, other relatives or nonrelatives in cities or towns for a long time because their mothers, fathers or both parents leave for work or study for over a half year and cannot take care of them $[12,13]$. Such group of children are not poor and some are even affluent; however, they feel loneliness and emptiness due to lack of communication and exchange with their parents and are highly susceptible to deviations in personality and behavioral patterns under temptations from complex environments.

Internet addiction refers to loss of control over impulsion for surfing the internet in the absence of addictive substances, representing a compulsive behavior similar to gambling [14]. Adolescents are high-risk group of Internet addiction, and susceptible age to Internet addiction is between ages of 13 and 18 in China [15]. In general, occurrence rate of Internet addition is $6 \%-14 \%$, while relevance ratio of urban left-behind children to Internet addiction is $6.83 \%$ [16]; therefore, it cannot be ignored that Internet addiction causes negative effects on urban left-behind children.

According to relevant studies [17-19], Internet addicts give preference to emotional words, especially to negative emotional information. A Study reported by Wu and Zheng [19] also indicates that Internet-addicted teenagers give preference and attention to negative emotional information. Therefore, the goal of this study was to discuss implicit preference in facial expression recognition of urban Internetaddicted left-behind children through adopting variant GO/NO-GO (GNAT) paradigm of implicit association test (IAT).

*Corresponding author: Ying Ge, Faculty of Psychology, Key Laboratory of Personality and Cognition, Ministry of Education, Southwest University, Beibei, Chongqing, China, Tel: +86 18623360139. E-mail: gy8620@163.com

Received April 18, 2014; Accepted July 21, 2014; Published July 23, 2014

Citation: Ge Y, Zhang J, Hu Y (2014) Study of Implicit Preferences in Facial Expression Recognition of Urban Internet-Addicted Left-Behind Children in China. J Biosafety Health Educ 2: 117. doi:10.4172/2332-0893.1000117

Copyright: @ $2014 \mathrm{Ge} \mathrm{Y}$ et al. This is an open-access article distributed under the terms of the Creative Commons Attribution License, which permits unrestricted use, distribution, and reproduction in any medium, provided the original author and source are credited. 
Citation: Ge Y, Zhang J, Hu Y (2014) Study of Implicit Preferences in Facial Expression Recognition of Urban Internet-Addicted Left-Behind Children in China. J Biosafety Health Educ 2: 117. doi:10.4172/2332-0893.1000117

Page 2 of 5

\begin{tabular}{|c|c|c|c|c|c|}
\hline Step & Experiment frequency & Practice/test & Q Response circumstance & P Response circumstance & Negative picture \\
\hline 1 & 20 & Practice & Positive picture & Non-ego word \\
\hline 2 & 20 & Practice & Ego word & Negative picture + non-ego word \\
\hline 3 & 20 & Practice & Positive picture + ego word & Negative picture + non-ego word \\
\hline 4 & 40 & Test & Positive picture + ego word & Positive picture \\
\hline 5 & 20 & Practice & Negative picture & Positive picture + ego word \\
\hline 6 & 20 & Practice & Negative picture + non-ego word & Positive picture + ego word \\
\hline 7 & 40 & Test & Negative picture + non-ego word & \\
\hline
\end{tabular}

Table 1: Steps of Implicit Association Test.

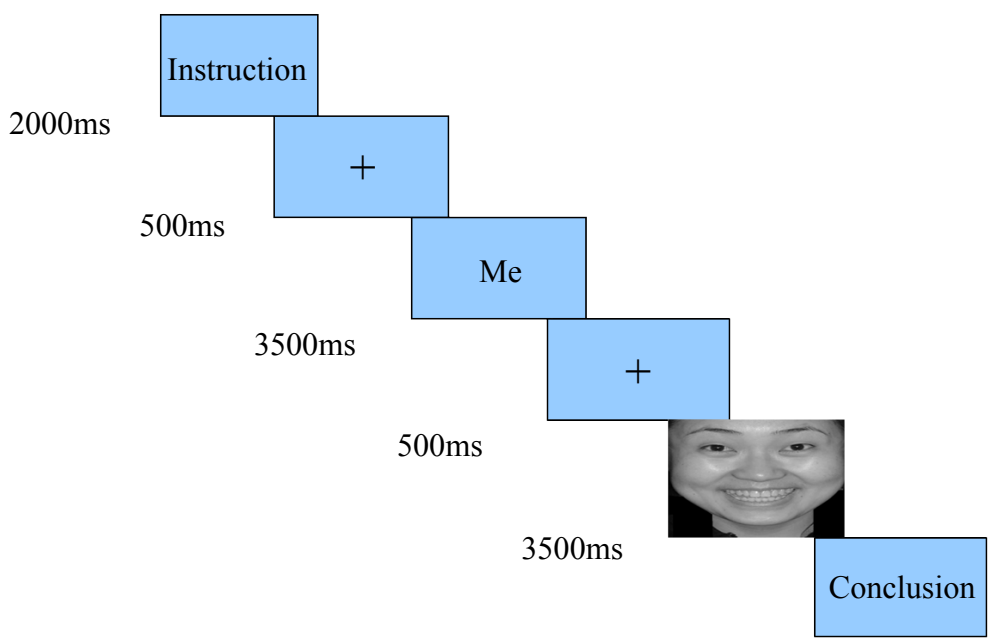

Press key and the

picture will disapper

Figure 1: Formal experiment flow chart.

\section{Method}

\section{Participants}

Clue sampling was adopted in this study. Through mental health surveys in two middle schools with large proportions of urban leftbehind children, we found that there existed internet use deviations among the students. Later than, we took children, below 18 years old, who, with registered permanent residences in cities or towns, are brought up by others because their mothers, fathers or both parents leave for work for over a half year as standard candidates for participants, conducted preliminary screening on urban left-behind children and selected out 500 urban left-behind children as the participants. Then, about one hour of interview was carried out aiming at the 500 children, and secondary screening was also conducted as per standards in Adolescent Pathological Internet Use Scale [20].

The scale has 38 questions in 6 dimensions: salience, social comfort, tolerance, compulsive surfing of the internet, withdrawal symptom and negative consequence. The scale adopts 5-point self-rating scale, ranging from "absolute inconformity" to "absolute conformity". With KMO index of 0.940 and Cronbach's Alpha coefficient of 0.948 , the scale possesses relatively high reliability and validity. Participants with average item score no less than 3.15 points were defined as "Internetaddicted individuals", while those with average score less than 3.15 points were defined as "non-addicted individuals". Finally, 30 urban left-behind children with Internet addiction and 30 urban left-behind children without Internet addiction (normal control group) were selected respectively as participants.

All participants were right handed with normal vision (with or without glasses) and euchromatopsy and without physiological maladies (such as heart disease and hypertension) or familial psychiatric history. The participants participated in the experiment voluntarily. Since the participants were all juveniles, the investigators obtained approval from the school and a signed consent from guardians prior to carrying out the experiment. Each of the participants was rewarded with a gift worth RMB 15 after the experiment.

\section{Experimental design}

A single-factor 2 level (urban left-behind children with Internet addiction and urban left-behind children without Internet addiction) experimental design was adopted for this study and the discriminability index d' in signal detection theory was adopted as the dependent variable.

\section{Experimental materials and preparation}

The affective pictures in Chinese face affective picture system [21] were used for this study. Ten pictures representing each emotional nature (positive and negative) were selected, five with male faces and five with female faces, so there-were 20 pictures in all, which were identified as attribute type. Target type was confirmed as the following words in the experiment: 10 ego words: I, my, me. mine, myself, oneself, my own, one's own, self and own; 10 non-ego words: he, his, outsider, 
outsider's, other's, themselves, their own, they, their and other people. Then, the programs used for the implicit association test were developed with E-prime professional software (Psychology Software Tools, Inc., Pittsburgh, PA).

During the process of the experiment, quietness was maintained in the laboratory, and the experiment was carried out separately in the sound-proof cell. After knowing basic experimental requirements spoken by the experimenter, participants pressed keys and completed the test process independently according to experimental instructions. The experimental materials were presented by 19" flat-panel monitor with display refresh rate of $85 \mathrm{~Hz}$ and resolution ratio of $1024 \times 758$ pixels.

\section{Experimental procedures}

Implicit association test was divided into seven steps (Table 1 and Figure 1 show the experimental process):

Step one: to present positive pictures and negative pictures at random (10 of each). Participants were required to classify the presented pictures and press the key as soon as possible, pressing key "Q" when positive pictures are presented and key " $P$ " when negative pictures are presented (Practice).

Step two: to present ego words and non-ego words at random (10 of each). Participants were required to classify the presented words and press the key as soon as possible, pressing key "Q" when ego words are presented and key "P" when non-ego words are presented (Practice).

Step three: to present positive pictures and negative pictures (5 of each) and ego words and non-ego words (5 of each) at random. Participants were required to press the keys as soon as possible, pressing key "Q" when ego words and positive pictures were presented and not pressing any key when non-ego words and negative pictures were presented (Practice).

Step four: to present positive pictures and negative pictures (10 of each) and ego words and non-ego words (10 of each) at random. Participants were required to press the keys as soon as possible, pressing key "Q" when ego words and positive pictures were presented and not pressing any key when non-ego words and negative pictures were presented (Test).

Step five: to classify positive pictures and negative pictures presented at random. Participants were required to press the keys as soon as possible, pressing key "P" when positive pictures were presented and key "Q" when negative pictures were presented (Practice).

Step six: to present positive pictures and negative pictures (5 of each) and ego words and non-ego words (5 of each) at random. Participants were required to press the keys as soon as possible, pressing key "P" when non-ego words and negative pictures were presented and not pressing any key when ego words and positive pictures were presented (Practice).

Step seven: to present positive pictures and negative pictures (10 of each) and ego words and non-ego words (10 of each) at random. Participants were required to press the keys as soon as possible, pressing key " $\mathrm{P}$ " when non-ego words and negative pictures were presented and not pressing any key when ego words and positive pictures were presented (Test).

\section{Pre-experiment and data processing}

Four middle school students were selected at random as participants of pre-experiment to demonstrate that the initial experimental design was favorable and can be directly applied in the formal experiment.

Data were combined by Emerge and then imported to SPSS13.0; gibberish was eliminated, leaving only correct response data in step four and step seven. Then, statistics was created on number of correct responses and wrong responses of each participant to different stimuli in step four and step seven. Thus, response matrixes of stage four and stage seven were obtained. d' of signal detection theory was acquired with the method below. Finally, comparison was performed on d'-score in both stages.

Analyze the two matrixes respectively:

1. with the matrix of stage four, positive d' to oneself and negative d' to others-were obtained. Method for obtaining positive d' to oneself: calculate false alert rate and hit rate with number of false alerts equal to number of error items (oneself + negativity) and hit number equal to number of correct items (oneself + positivity), and then translate false alert rate and hit rate into Z-score; Z-score of hit $-\mathrm{Z}$-score of false alert $=$ positive d' to oneself. Method for obtaining negative d' to others: calculate false alert rate and hit rate with number of false alerts equal to number of error items (others + positivity) and hit number equal to number of correct items (others + negativity), and then translate false alert rate and hit rate into Z-score; Z-score of hit - Z-score of false alert $=$ negative d' to others.

2. With response matrix of stage seven, negative d' to oneself and positive d' to others are obtained. Method for obtaining negative d' to oneself: calculate false alert rate and hit rate with number of false alerts equal to number of error items (oneself + positivity) and hit number equal to number of correct items (oneself + negativity), and then translate false alert rate and hit rate into $\mathrm{Z}$-score; Z-score of hit $-\mathrm{Z}$-score of false alert $=$ negative $\mathrm{d}$ ' to oneself. Method for obtaining positive d' to others: calculate false alert rate and hit rate with number of false alerts equal to number of error items (others + negativity) and hit number equal to number of correct items (others + positivity), and then translate false alert rate and hit rate into Z-score; Z-score of hit - Z-score of false alert $=$ positive d' to others. Finally, import all the data above into SPSS13.0 and carry out independent samples t-test to get final results.

\section{Results}

After getting d' upon data processing, results (Table 2) were obtained through analysis on d' (implicit preference $=$ positive $\mathrm{d}^{\prime}-$ negative d'). Through independent samples t-test on participant type, it was found that there was very significant difference between the two groups of participants on positive attitude to oneself $(p<0.01)$, addicted group lower than non-addicted group; there was significant difference between the two groups of participants on negative attitude to oneself ( $p<0.05$ ), with addicted group higher than non-addicted group; there was significant difference between the two groups of participants on implicit preference to oneself $(p<0.05)$. However, there was no significant difference on positive attitude, negative attitude and implicit preference for others.

\section{Discussion}

d' index reflects preference to some kind of emotional pictures; d' of participants for unified task of ego words and positive emotional pictures is higher than that for unified task of ego words and negative 


\begin{tabular}{|c|c|c|c|c|}
\hline & Type of participant & Positivity & Negativity & Implicit preference \\
\hline & Non-Internet addicted & $2.26 \pm 0.62$ & $0.96 \pm 1.25$ & $1.31 \pm 1.32$ \\
\hline \multirow[t]{3}{*}{ Oneself } & Internet-addicted individuals & $0.92 \pm 1.46$ & $2.39 \pm 1.17$ & $-1.40 \pm 1.33$ \\
\hline & $t$ & $2.83^{\star \star}$ & $-2.77^{*}$ & $4.85^{\star}$ \\
\hline & Non-Internet addicted & $1.11 \pm 0.74$ & $2.06 \pm 0.87$ & $-0.96 \pm 1.38$ \\
\hline \multirow[t]{2}{*}{ Others } & Internet-addicted individuals & $0.85 \pm 1.14$ & $1.18 \pm 1.36$ & $-0.32 \pm 1.95$ \\
\hline & $t$ & 0.62 & 1.84 & 0.36 \\
\hline
\end{tabular}

Table 2: Sensitivity Index ( $\left.d^{\prime}\right)$ and Difference Test.

pictures, which indicates that participants have implicit preference for positive emotional pictures, and vice versa [22]. This study results showed that the non-addicted group of urban left-behind children had implicit preference for positive emotional pictures, while the Internetaddicted group of urban left-behind children had implicit preference for negative emotional pictures, i.e. compared with non-addicted urban left-behind children, Internet-addicted urban left-behind children preferred negative emotions more. Implicit preference can reflect attitude towards oneself and others. In terms of attitude towards oneself, the non-Internet addicts behaved more positively while the Internetaddicted individuals behaved more negatively. Left-behind children may have problems due to specific growth environment: insufficient home education, indifferent family relations, insufficient guidance or excessive interference, protection or punishment given by parents or guardians to the children, and so on [23]. When all these problems cannot be appropriately solved, negative emotion of children will be increased, and when the negative emotion is increased to a certain level, many children will choose to surf the Internet to get rid of negative emotions; thus, they develop cognition-behavior model of pathological internet use and have many inappropriate negative cognitive schema [24]. Consistency of external stimulus and existing cognitive schema makes information processing easier [25]. As for Internet addicts, selection bias of individuals to emotional information was guided by negative schema in the brain, which decreased their positive evaluation of themselves and made them have implicit preference for negative emotions.

\section{Conclusion}

The results of this research suggest that compared with urban nonaddicted left-behind children, urban internet-addicted left-behind children had more preference for negative emotions and poorer evaluation on their egos.

\section{Suggestions and Countermeasures}

Urban left-behind children are in the critical periods of physical and mental development and formation periods of their views of life, world views and values, and they greatly need attentions and guidance from adults. However, due to the particularity of their growing environments - their parents do not live together with them, when they face any problem that cannot be solved timely, they can only vent their negative induced emotions and handle their emotional disturbances by virtue of cyber world, which will finally make they rely on internet network and have instable emotions, anxieties, uncommunicative and eccentric dispositions and other negative personality characteristics. Aiming at the aforesaid current situation of the urban left-behind children, the following aspects shall be well done:

\section{Establish good parent-child relationship}

Although parents of the urban left-behind children work in other places, they shall often communicate with their children and care for their psychological needs. Moreover, they shall return homes at holidays and festivals to accompany with their children, and communicate with the children in a heart-to-heart way and let the children feel concerns and warmth from families.

\section{Strengthen school education responsibilities}

Firstly, schools and teachers shall show more concerns on the unban left behind children and shall exchange with the children's parents or guardians more and cooperate to educate the children well. Secondly, schools shall strengthen education on internet morality, train correct view of internet of the urban left-behind children and enhance educators' internet qualities to guide internet behaviours of the urban left-behind children. Finally, schools shall strengthen campus network construction to build a civilized internet environment. As for urban left-behind children who have problems, schools shall ask them to go to psychological counselling rooms to tell their confusion and troubles to psychological counselling teachers and let the teachers help them solve their problems and guide their healthy psychological development.

\section{Optimize social internet environment}

First of all, monitoring towards illegal and harmful information of internet network shall be enhanced, so as to create a good cyberspace environment for urban left-behind children. In the second place, social attentions shall be paid to internet-addicted urban left-behind children because they not only need material satisfaction, spiritual fulfilment but also need the society to guide their ways out of internet addiction.

\section{Declarations of interest}

All authors declare no conflicts of interest.

\section{Acknowledgement}

This work was supported by the China, Chongqing Social Science Planning Project (2010YBJY40).We thank the many students from Zhongshan Middle School and Wolong Middle School. Finally, we thank the anonymous reviewers for their thoughtful comments.

\section{References}

1. Nosek BA, Banaji MR (2001) The Go/ No-go Association Task. Social Cognition 19: $625-664$

2. Chengrong D, Fulin Z (2005) A Study on Current Situation of Left-behind Children in China. Population Research 1: 29-30.

3. Fulin Z, Chengrong D (2006) Survey on Studies on Left-behind Children Population Journal 3: 62-65.

4. Zhen H, Lijuan C (2007) Research on Defining the Standards of Left-behind Children. China Youth Study, 10: 40-43.

5. Chengrong D, Ge Y, Ying W (2008) A Study on Current Situation of Rural Leftbehind Children in China. Population Research 5:15-25.

6. Jing L, Wei W, Wenbin G (2009) Review of the Studies on Rural Left-Behind Children in China. Advances in Psychological Science 17: 990-995.

7. Xinghua F, Xiaoyi F, Qinxue L, Yang L (2009) A Social Adaptation Comparison of Migrant Children, Rear Children and Ordinary Children. Journal of Beijing Normal University (Social Science Edition), 5: 33-40. 
Citation: Ge Y, Zhang J, Hu Y (2014) Study of Implicit Preferences in Facial Expression Recognition of Urban Internet-Addicted Left-Behind Children in China. J Biosafety Health Educ 2: 117. doi:10.4172/2332-0893.1000117

Page 5 of 5

8. Jingxin Z, Xia L (2010) Rural Left-Home-Children's Depression and Antisocial Behavior: the Protective Role of Daily Pleasures. Psychological Development and Education 6: 634-640.

9. Hui L (2012) The Resilience and Adaptive Research on the Children Left Behind - Take the Children Left Behind in Changyang Tujia Autonomous County as an Example. Master Thesis. Wuhan: South-Central University for Nationalities.

10. Lin L (2008) The Study of Use Blog to Solve the Problems in Family Education of City Left Behind Children. Master Thesis. Changchun: Northeast Normal University.

11. The Education, Science, Culture and Health Committee of Chongqing Municipal People's Congress. (2012) Investigation Report on Education and Cultivation Situation of Rural Left-behind Children in Chongqing. Bulletin of Standing Committee of Chongqing Municipal People's Congress.

12. Zhi W, Qi J, Dajun Z (2008) A Study on Coding and Recognition of Internet Addicts. Psychological Development and Education 24: 106-112.

13. Dan Z, Yanjun C, Deng Jun D (2012) Effect of Family Education on Urban Left-behind Children and Countermeasure Analysis. Mental Health Education in Primary and Secondary Schools 11: 21-23.

14. Young KS (1998) Internet addiction: the emergence of a new disorder. Cyber Psychology and Behavior 3: 237-244.

15. Wenbin G, Zhiyan C (2006) A Study on Psychopathology and Psychotherapy of Internet Addiction. Advances in Psychological Science 14: 596 - 603.

16. Cancan J, Zhiyong Q, Xiaohua W (2010) On the Current Situation of Internet Addiction of Left-at-home Children and Migrant Children, Their Mental Health and Interpersonal Relationships. Chinese Journal of Special Education 7: 5964.
17. Riesenhuber M (2004) An action video game modifies visual processing Trends in Neuroscience 27: 72-74.

18. Shujuan L (2005) Research on Selective Cognitional Bias to Internet Related Stimuli in Heavy Internet Users. Master Thesis. Hangzhou: Zhejiang University.

19. Wenli W, Xifu Z (2012) Characteristics of Information Selection in Pathological Internet Adolescent Users. Chinese Mental Health Journal 26: 631-634.

20. Li L, Yang Y (2007) The Development and Validation of Adolescent Pathological Internet Use Scale. Acta Psychologica Sinica 39: 688-696.

21. Xu G, Yuxia H, Yan W, et al. (2011) Revision of the Chinese Facial Affective Picture System. Chinese Mental Health Journal 25: 40-46.

22. Ying Z, Junsheng L (2009) Using Eye-movement to Study Implicit Aggressiveness. Psychological Science 32: 858-860.

23. Min D, Xu C, Xuefeng Z, et al. (2010) Moderating Effects of Alienation between Coping Style and Social Adjustment for Left-behind Middle School Children. Chinese Journal of School Health 10: 1185-1187.

24. Davis RA (2001) A cognitive-behavioral model of pathological Internet use. Computers in Human Behavior 17: 187-195.

25. Beck AT, Clark DA (1988) Anxiety and depression: An information-procession Perspective. Anxiety Research 15: 23-26. 